\title{
Development of MEMS Angular Rate Sensor with Self-calibration Function
}

\author{
S. Timoshenkov, N. Korobova*, A. Mikheev, A. Timoshenkov \\ Microelectronics Department \\ National Research University of Electronic Technology (MIET) \\ Zelenograd, Moscow, Russian Federation \\ *korobova3@mail.ru
}

\begin{abstract}
In the paper, new principles and methods for determining the transfer characteristic parameters for a micromechanical angular velocity sensor, as well as an assessment of the significance and efficiency of these parameters, have been described. The structure and algorithms for obtaining and processing calibration coefficients for implementing the MEMS self-calibration functions have been developed. The possibilities and degree of automation of processes for obtaining individual characteristics, testing and calibration have been determined.
\end{abstract}

Keywords-angular velocity sensor; design, signal, transfer characteristic.

\section{INTRODUCTION}

The publication analysis of micro system technology shows that at present, there are basic directions that can be defined as the development of: a) sensors and activators, b) energy micro machines, c) micro-robot systems, d) analytical and technological Microsystems. Fabricate sensor devices necessary to use the modified technological techniques of microelectronics. This makes it possible to produce precision unique products which dimensions and mutual arrangement of elements are reproduced with micron and submicron accuracy [1-3].

Inertial micromechanical sensors (IMMS) are very widely used in modern technology. Functionality, typologies and quality criteria of these products are greatly expanding with the growth of application areas and the market. For consumers, it is sometimes difficult to compare instruments objectively. There are different points of view on the quality criteria of the IMMS even among developers and manufacturers. The fundamental and basic characteristic of any sensor is the transfer characteristic, which is the dependence describing the relationship between the input and the output signals. For a detailed description of the IMMS limit accuracy [2], along with the definition of the transfer characteristic, attention should be paid to long-term drift analysis and the identification of a systematic and random error component. An angular velocity sensor (AVS) is designed to measure object angular velocity. It can be used in systems operating at high overloads [3].

This work was supported by the Russian Science Foundation. Unique identifier for applied research is 16-19-00177
Production lines equipment of various sensor manufacturers is mainly represented by equipment that allows setting control actions with high accuracy [4]. Equipment, simulating various effects, was used most often in the manufacture and calibration of various MEMS sensors and systems. Test complexes are built around a specific impact simulator and most often consist of different blocks of analog-to-digital converters (when manufactured systems have an analog signal) and converter interfaces that allow one to receive and process information from sensors and systems in digital form [5]. The model of the impact simulator, dimensions of tested MEMS sensors and equipment determine the automation degree of tests [6]. Some firms implement a special rig that allows one to test simultaneously several axes of sensors or systems on a single-axis stand. Obvious advantage of this method is the reduction of testing time due to the simultaneous testing of several axes. However, this method cannot produce results that are more accurate and requires the development of special algorithms for processing results. Another disadvantage of this method may be complexity of manufacturing tooling, directly affecting the accuracy of the produced calibration. In the paper, some new principles and methods for determining the transfer characteristic parameters for the micromechanical angular velocity sensor have been described, as well as an assessment of the significance and efficiency of these parameters. Another promising direction for scientific development is related to the methods of integrating signals from sensors that allow increasing the received data accuracy, as well as partially compensating "individuality" of the sensor characteristics [7]. The appearance of individual characteristics arises from defects and errors during sensitive elements installation on the printed circuit board, from possible deviations in the layout of the printed circuit board in the housing, its bends, solder defects, conductors, scatter of the element parameters, etc. The carried out work made it possible to outline a promising direction for further investigation associated with the development and methods improvement of the complexion signals from several sensitive MEMS elements. The authors' studies are related to both different types of sensing elements and their location within the sensors or the inertial system. Our motivation was also additional experiments to obtain and to use new calibration techniques. The results may allow one to increase 
and to improve parameters of MEMS sensors and systems, developed on their basis.

\section{EXPERIMENTAL PART}

Deep etching silicon elements of MEMS capacitive accelerometers were carried out on I-Speeder (Alcatel). The authors used silicon wafers with thickness $300 \mu \mathrm{m}$ and (100) orientation of wafer surfaces. Bonding silicon with glass was carried out on SB6 Carl Seuss. The authors used different climatic (Espec Corp MC-811P) and mechanical (LDS V455 with PA 1000L and Acutronic 1135S) devices [8] for measurement the considered parameters. The automatic test bench for testing the self-calibration algorithms consisted of the AcutronicAC1120S rotary table, the MOXAUPORT 1610$B$ interface converter, the National Instruments USB-6251 external ADC, GWinstek PSP-2010 power supply and a computer with the control program.

MEMS consists of a sensing element and a digital part that provides of reception, processing and filtering signals from the sensitive part, output signal formation, and data exchange implementation via RS-232 interface. Sensor will output analog signals corresponding to three sensitive sensor axes, temperature information and duplicate this information digitally. A specially implemented command system and a data exchange protocol will allow one to obtain full information about sensor readings via the RS-232 interface, and also to translate the sensor into the service mode, necessary for its calibration and tuning. Realized data exchange between MEMS sensors and test stand will significantly simplify a number of technological operations related to the adjustment of zero sensor signals, scale factors installation, calibration coefficients, etc. The realized protocol of production tests will make it possible to estimate and analyze the non-orthogonality of measuring axes based on the information received from the sensor, and to detect the nonlinearity of scale factors.

The developed test program involves obtaining output values using analog and digital interfaces at control points throughout the measuring sensor range.

Measurement of the inertial sensor transfer characteristic in the classical understanding presupposes setting a number of standard effects, recording the output signal with known input data, and analyzing the dependence of the output signal on the input effect. Traditionally, this dependence is approximated to a linear function and two coefficients are determined:

(1) "scale factor" (tangent of the transfer characteristic slope) and

\section{(2) "zero signal" (free term of the linear dependence).}

Zero signal value is very close in meaning to the sensor value with zero input effects, but in general it is not synonymous. From the user's point of view, reliable knowledge of the transfer characteristic steepness or scale factor is necessary. There is often a dual understanding with respect to the free term of the transfer function or zero signal (or sensor at rest indication). The importance and practical significance of these parameters strongly depend on the specific application. Figure 1 shows the transfer characteristic of AVS with linear interpolation of 7 experimental points. As can be seen from the graph (Figure 1), sensor output signal (digital) $\mathrm{D}$ can be best described by the equation

$\mathrm{D}=\mathrm{k} \omega+\mathrm{b}$

where $\omega$ is the angular velocity measured in degrees per second; $\mathrm{k}$ - scale factor was -394.8426 and was measured in [(units of code) / (degrees per second)]; b - t zero signal was 32879 and was measured in output code units.

The initial data for calculating these transfer characteristics were as follows: at 7 different angular velocities (-30, -20, -10, $0,10,20,30$ degrees per second) the output signal were averaged as


code), respectively.

The error in angular velocity setting can be neglected taking into account the averaging time of signal and turntable accuracy. If we substitute the values of the acting angular velocity into the just-defined transfer function (equation 1), then the output signal values (D), which are on the same straight line and differ from the actual ones will be obtained.

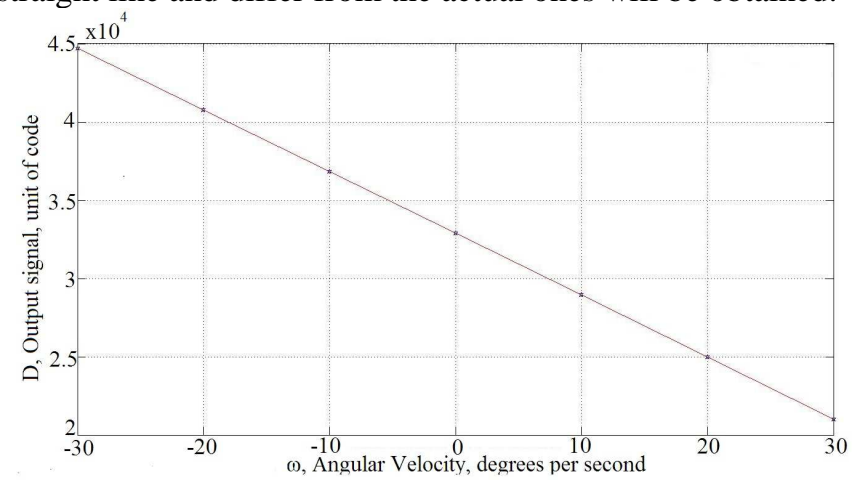

Fig. 1. AVS transfer characteristic with linear interpolation of experimental points

The best conformity was achieved by interpolating the transfer characteristic values by the 6-th order polynomial:

$$
\begin{array}{llllll}
40776 & 36827 & 32879 & 28931 & 24982 & 21034
\end{array}
$$

Difference between actual values of the output signal and the values at the output that are offered to the linear transfer function (series of numbers 2) indicates the nonlinearity of the transfer characteristic or the relative error in the measuring range. If the researcher has the ability to set the angular velocity, considering it as a standard velocity and to calculate the nonlinearity with respect to it, then the consumer on the object has only the sensor output signal and the transfer characteristic is represented as $\omega=f(D)$. The authors present dependence $D=f(\omega)$ (1) in the form of $\omega=f(D)$, which is more convenient for the majority of consumers, then:

$$
\omega=-0.0025 \cdot \mathrm{D}-(-83.2710)
$$


where $\omega$ is the angular velocity, D is the output code of sensor. The authors obtain the following transfer characteristics substituting the actual AVS values, obtained in the experiments:

$\begin{array}{lllllll}-29.9663 & -19.9891 & -10.0061 & -0.0486 & 9.9573 & 19.9989 & 30.0539\end{array}$

In the first considered example (first point), the nonlinearity will be for $\omega$ (D) dependence:

$$
|((-30-(-29.9663)) / 60) \cdot 100|=0.0562 \%
$$

The value for all 7 experimental points was calculated and such series was obtained as:

$$
\begin{array}{lllllll}
0.0561 & 0.0182 & 0.0101 & 0.0810 & 0.0712 & 0.0018 & 0.0898
\end{array}
$$

The maximum value in the 7-th point of the experimental data was mismatched with the approximating function at $0.0898 \%$ of the measuring range (Figures 2-3).

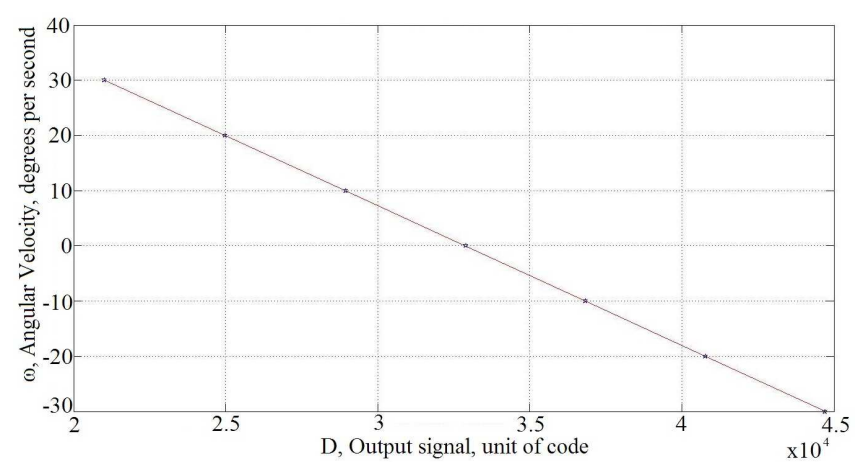

Fig. 2. Experimental data and linear approximation of the transfer function (D)

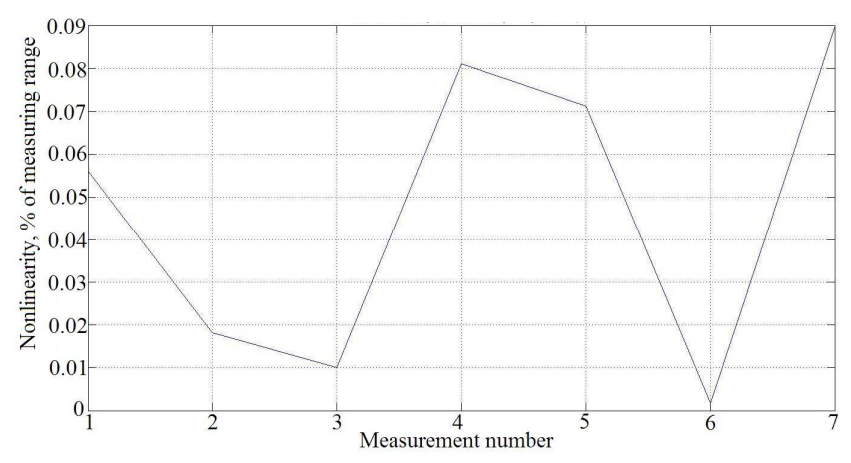

Fig. 3. Nonlinearity of the transfer characteristic for AVS

\section{RESULTS AND DISCUSSION}

Early models of testing equipment for precision MEMS sensors expected individual and manual processing of data that was received during the sensor test. More time for operations to change sensor orientation would spend. The testing process involves the joint work of a two-people team (an operator and a specialist). The main operator's tasks were to control the measuring equipment and to set the control actions for each of the sensor axes in a certain test sequence. To do this, it was necessary to install the sensor in a certain way on the test equipment (turntable), using the control interface for the turntable, to set the control effect from the list and to record the digital and analog sensor signals. The result of the sensor tests was data array, containing the measurement results of analog and digital signals, as well as a long record of the output signals, which allow determining the parameters of instability and signal drift. After the formation of the data set by the operator, the work of the specialist begins. Calculations are made to obtain the main characteristics of the sensor using a set of programs, as well as to draw conclusions about the need to correct some parameters and the need for repeated testing.

Such test procedure requires a lot of time for the operator and the specialist of high qualification, the need to train the operator to work with various scientific equipment, accuracy and care when following the test program. The large amount of manual labor and the operator involvement in many technological operations during the test led to numerous errors at various stages and made the testing and production process more difficult, allowing evaluating the characteristics of individual sensors and making it difficult to produce even small batches of MEMS sensors.

The nonlinearity of the transfer characteristic has a clear meaning in estimating the straightness and repeatability of the transfer characteristic. For modern AVS, the normal level of maximum nonlinearity lies in the measuring range of $0.01 \ldots .0 .5 \%$. The nonlinearity of the transfer characteristic with a less than $0.01 \%$ level, when translated into absolute units, will show the level of the random component or noise. A specific value of a zero signal, defined in the transfer characteristic composition for some consumers of inertial sensors, is not at all fundamentally important. Indeed, often the zero-signal shift in the entire temperature range is much more important than measuring the steepness of the transfer characteristic. The specificity of some applications allows the system to calibrate the zero signals every time directly in a dormant operating environment. The drift level of a zero signal, its stability from "switching on" to "switching on", and many other sensor parameters are of great importance.

It is absolutely impossible to satisfy a consumer without an accurate determination of the scale factor. If it is not necessary for the consumer to determine both the scale factor and the zero signals as a result of experiment interpolation at several angular velocities, then there is a much simpler way of determining the scale factor of the AVS. If one rotates the AVS in a single plane for a short time by a certain amount, it is easy to calculate the integral of the output signal with respect to the signal at rest.

It is easy to choose the ratio between the output signal and the actual angular velocity knowing the angle of sensor 
rotation. Such simplified approach can make sense if the sensor has a relatively good linearity (not more than $0.3 \%$ ) and the measurement range is not very large so that the rotation velocity in the experiment will be close to the typical sensor velocity. The obvious advantages of this method are fast and simple, including the absence of the need for precise rotary mechanisms. To specify a unique angular position, for example, full 360 degree rotation, one can use the most primitive snap (Figure 4).

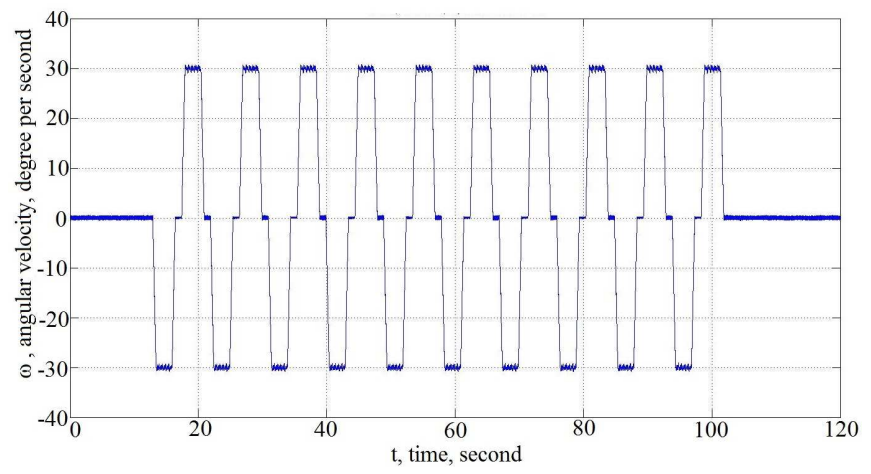

Fig. 4 Initial data for determining the scale factor by integration (10 rotations by 90 degrees).

Experiments were carried out for evaluation of comparative methods on the AVS of the TG-75 series. Based on a series of 10 experiments aimed to determine the scale factor by the classical method, i.e. by sequentially setting the angular velocities and interpolating the dependence to a linear function, and a series of 10 experiments aimed to determine the scale factor through the time integral, one can conclude that the scale factors measured by the two methods are practically equal. They had a negligible difference, commensurate with standard deviation from experiment to experiment. Data spread from experiment to experiment was 1.5 times greater in the integral-based method, but this method is at least 3 times faster, less labor-intensive, and requires minimal equipment.

There are following results of the development of key assembly processes for ring resonant micromechanical element of the MME gyroscope [8]. The developed assembly technology consists of two main stages: the first stage assembly of the sensor chip in accordance with Figure 5, the second stage - encapsulation and vacuum of sensor chip in the housing. Silicon sensitive element (1) was connected to the glass base (2) by the anode splice method. In this case, base glass composition was chosen such that the coefficients of thermal expansion of the glass and silicon practically were coincided. Sensitive element resonator remains suspended in a magnetic field formed by the magnet (3) and the upper (4) and lower (5) poles made from soft magnetic material. The magnetic circuit was designed in such way as to form the maximum magnetic field in the resonator zone (and, consequently, the conductors located on the resonator surface). Thus, it was possible to improve thermal stability of gyro operation and to increase its sensitivity.
The technological scheme of the sensor assembly has been developed based on these studies and shown in Figure 6.The assembly begins with the lower magnet pole planting to the base, using the solder glass.

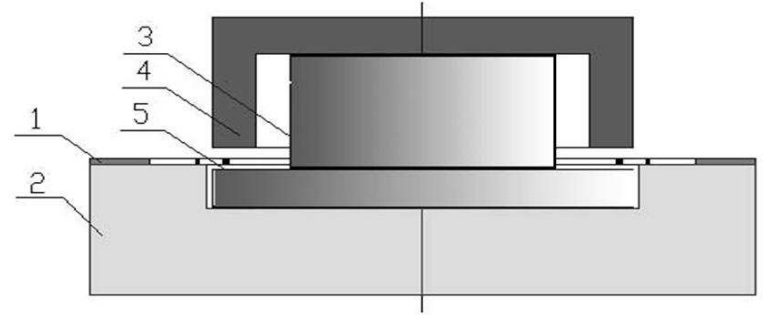

Fig.5. A general view of the ring micromechanical gyroscope chip: 1 silicon element; 2 - glass base; 3 - magnet; 4 - upper pole of magnet; 5 - lower pole of magnet

Control parameters of the conductive circuits continuity have been made after anodic splicing of the sensor and the base.


gyro

Fig.6. Technological scheme of sensing element assembly for ring type

Then adhesive landing on the magnet and the upper magnet pole consistently was made. This completes the fabrication of the sensor chip. The chip on a glass plate was placed on the housing base with a POIn-50 solder. The next step was the creation of microchip interconnects with the base. Pins unwelding was carried out by ultrasonic contact welding and aluminum or gold wires. "Metal - glass" housing with a formed hole was sealed by laser welding. Locally melting solder in the housing hole by infrared heating in a vacuum has been produced after thermal degassing. The housing then was gradually cooled (to avoid thermal shock) and taken out from the vacuum chamber after depressurization [9]. All operations were performed on standard equipment in accordance with the developed technology. The process of applying a thick oxide layer $3 \mu \mathrm{m}$ thick, which was used as a masking coating, and 
anisotropic etching of silicon to a depth of $100 \mu \mathrm{m}$, was carried out on a Plasmalab-80 laboratory unit.

After that, the testing of the sensing element has been carried out. The designed system for signal removing is placed on the PCB. The sensitive element and the circuit board have been placed in the designed and manufactured gyroscope housing. Studies of gyro characteristics were undertaken. Special equipment providing a quick and easy change for the sensor axes for simultaneous testing of sensor groups was implemented. The developed software allowed almost completely automating the process of sensor testing, the formation of measurements archive, the application of various control actions for sensor testing, calculations and obtainment of the necessary data and calibration coefficients. New software allowed reducing the requirements for the operator qualification. All the necessary operator actions in the work process were displayed on the computer screen. It was necessary to enter the serial number, model and range of the sensor to determine the test parameters before the tests began. Next, it was necessary to change the sensor orientation relative to the turntable during the measurement on the software demand. The introduction of new software and design of the test stand allowed one to increase the test line productivity 2.5 times, to simplify significantly the testing process and almost completely eliminate the human factor in the errors when testing the sensors. It is planned to work out the possibility of automatic sensor orientation change, which will allow for complex and long-term testing without operator involvement. The developed test procedure allowed efficient and accurate calculation of correction factors for each tested inertial measuring unit. A stand can have simultaneous testing of five MEMS sensors or systems based on them simultaneously in automatic or semi-automatic mode. The conducted MEMS sensor tests showed that the procedure for checking the sensor readings at control points in the whole range of measured effects allows one to determine the appearance of nonlinearity in scale factors. In the case of testing new sensing elements or signal processing algorithms for the digital sensor part, more thorough testing with a large number of control points in the measured range of effects for the sensor is necessary. However, to test the efficiency of algorithms and constructions that have already been worked out, it is possible to reduce the number of control points, which allows reducing the testing time of the sensor, while retaining the ability to determine anomalies or malfunctions in the test samples. Test procedure has been developed in a certain temperature range to determine the temperature errors. Deviations in scaling factors and zero sensor signals were detected at the extreme values of the temperature range.

\section{CONCLUSION}

Moore's Law greatly influenced the development of semiconductor electronics by setting a high growth rate of its most important component - microelectronics. Subsequently, it contributed to the emergence and development of new areas, for example, micro-system technology in the form of MEMS and nano-electronics [10]. As a result, electronics has become the leading sector, largely determining the development of the world economy as a whole. To a large extent, the conventional electronic component technologies exhausted their potentialities due to the fundamental limitations of the properties of materials. The problem is not so much the inability to produce thin functional layers of the desired quality, but the fact that the structure of the conventional semiconductors not allows to improve the integration of microelectronic elements and to reduce their size to the extent necessary. Therefore it is necessary to look for not only new materials but for new engineering solutions.

The performed works and study of the MEMS sensor models characteristics allowed us to outline a promising direction for further research related to the development and improvement of the methods for combining signals from several sensitive MEMS elements, and also related both to different types of sensing elements and their arrangement within the sensors or the inertial system. The received and practically confirmed data, supplemented by experiments on obtaining and using new calibration methods, can allow increasing and improving the parameters of MEMS sensors and systems developed on their basis.

The presented studies make it possible to quickly measure the scale factor through the angular velocity integral, which can be recommended in the mass AVS production. The specified characteristics of the devices, depending on the customer's requirements, can be adjusted in a fairly wide range. In reality, it is not so simple. Lowering production cost and searching for new consumers are persistent problems, with customers often agreeing to a reduction of price, even at the expense of some deterioration in the quality characteristics of ICs.

The main emphasis in the paper was made on the allocation and recording of all regular and nonrandom components of inertial sensor errors and separately on the work with random components of the output signal. Systematic error components, such as a zero signal or scale factor correction is the primary self-calibration of sensors implemented in the internal device calculator. A deeper aspect of self-calibration involves some actions that can partially reduce the effect of random components on the resulting sensor error.

There is a module in the structure of the test stand for analyzing the long-term drift of accelerometers and gyroscopes, using the Allan variation to isolate the random components of the micromechanical sensor output signal and to separate them into types. With this method, it becomes possible to separate and quantify the quantization noise, white noise (white noise spectral power or speed walk or random angular walk in the case of accelerometers or gyroscopes, respectively), minimum drift instability, temperature and a long-term trend of the output signal. Careful analysis of the output signal is necessary to reduce the random component. This is possible due to the use of information from several micromechanical gyroscopes. Comparative assessments of improving the micromechanical sensor accuracy in three types of experiments have been made: (1) due to the simple 
summation of signals from a group of sensors (and subsequent averaging), (2) by summation of pair wise differentially located gyroscopes, (3) due to adaptive (dynamic) Kalman filter.

The operation of a complex gyro, (consisting of a sensor series), was analyzed both in the static position and under dynamic conditions. The criteria for the joint estimation of the operation accuracy for a group of gyroscopes and its informativeness have been proposed.

A criterion of non-zero information of the sensor output signal has been proposed for automatic adjustment of the adaptive filter coefficients.

It is assumed that the internal calculator in the inertial module will be able to select the filtration coefficients, thus, sensor self-calibration can be performed at the level of longterm drift characteristics improving. The individuality of the sensor characteristics is the principle complexity in recording the signal from a group of sensors and when observing all random components of the sensor group.

\section{ACKNOWLEDGMENT}

This work was financially supported by the Russian Science Foundation. The unique identifier for applied research is $16-19-00177$.

\section{References}

[1] V.K. Varadan, K.J.Vinoy, K.A. Jose, RF MEMS and Their Applications, West Sussex: John Wiley\& Sons Ltd, 2003, 528p.

[2] V.Verner, E. Kuznetsov, A Saurov, "Fiftieth anniversary of Moore's law: development of micro- and nanoelectronics", Nanoindustry, vol.59, N5, pp.56-72, 2015.

[3] Nanotechnology in Electronics. Ed. Y.A. Chaplygin. Issue 3. Moscow: Technosphere, 2015, $480 \mathrm{p}$.

[4] S. Beeby, G. Ensell, M. Kraft, N. White, MEMS Mechanical Sensors, Artech House. Inc.: Boston, London, 2004, pp. 4-94.

[5] N. Lobontiu, E. Garcia, Mechanics of Microelectromechanical Systems, Springer, Kluwer academic publishers: Boston, 2005, pp. 263-286.

[6] S.A. Anchutin, E.S. Morozova, A.S. Golovan, V.F. Shilov. "System for initial angularmapview to the object, based on micromechanical accelerometers”, Russian J. Devices, №12, pp.58-60, 2010.

[7] Nanotechnology in Electronics. Ed. Y.A. Chaplygin. Issue 1. Moscow: Technosphere, 2005, $435 \mathrm{p}$.

[8] E. Kochurina, S. Timoshenkov, N. Korobova, Y. Chaplygin, S. Anchutin, A. Kosolapov, "Research and development of capacitive transducer with linear acceleration", Proceedings [SPIE Microtechnologies -2015, 4-6 Barcelona, 9517, 951725].

[9] A. Shalimov, S.Timoshenkov, N. Korobova, M. Golovinskiy, A. Timoshenkov, E. Zuev, S.Berezueva, A. Kosolapov, "Comb structure analysis of the capacitive sensitive element in MEMS - accelerometer", Proceedings [SPIE. Int, Soc. for Opt.Eng. January 2015, 9467, 94672W].

[10] Nanotechnology in Electronics. Ed. Y.A. Chaplygin. Issue 2. Moscow: Technosphere, 2013, $688 \mathrm{p}$. 